\title{
The Foundational Theory of Optimal Bayesian Pairwise Linear Classifiers
}

\author{
Luis Rueda ${ }^{1}$ and B. John Oommen ${ }^{2}$ \\ 1 School of Computer Science, Carleton University, 1125 Colonel By Dr., Ottawa, \\ ON, K1S 5B6, Canada. \\ \{lrueda, oommen\}@scs.carleton.ca \\ 2 Senior Member, IEEE.
}

\begin{abstract}
When dealing with normally distributed classes, it is well known that the optimal discriminant function for two-classes is linear when the covariance matrices are equal. In this paper, we determine conditions for the optimal linear classifier when the covariance matrices are non-equal. In all the cases discussed here, the classifier is given by a pair of straight lines which is a particular case of the general equation of second degree. One of these cases is when we have two overlapping classes with equal means, which is a general case of the Minsky's Paradox for the Perceptron. Our results, which to our knowledge are the pioneering results for pairwise linear classifiers, yield a general linear classifier for this particular case, which can be obtained directly from the parameters of the distribution. Numerous other analytic results for two and $d$-dimensional normal vectors have been derived. Finally, we have also provided some empirical results in all the cases, and demonstrated that these linear classifiers achieve very good performance.
\end{abstract}

\section{Introduction}

The aim of statistical Pattern Recognition (PR) is to find a discriminant function which can be used to classify an object, represented by its features, which belongs to a certain class. In most cases, this function is linear or quadratic. When the classes are normally distributed, it is not always possible to find the optimal linear classifier. In all the known results in this field, determining a linear function to achieve Bayes classification for normally distributed class-conditional distributions, has only been reported when the covariance matrices are equal [1].

As opposed to optimal linear classifiers, many attempts have been made to yield linear classifiers, using Fisher's approach [3], [2], 13, the perceptron algorithm (the basis of the back propagation Neural Network learning algorithms) [9], Piecewise Recognition Models [8], Random Search Optimization [10], and Removal Classification Structures [1]. All of these approaches suffer from the lack of optimality, and thus although they find linear discriminant functions, the classifier is not optimal.

In this paper, we show that there are other cases for normal distributions and non-equal covariance matrices in which the discriminant function is linear 
and the classifier is optimal. One of these cases is when we have two overlapping classes with equal means, and mutually orthogonal covariance matrices. But as opposed to all the previously studied linear classifiers, the new techniques introduced here yield pairwise linear classifiers, which emerge as degenerate cases of the general quadratic classifier.

Minsky showed that it is not possible to find a single linear classifier for the simple case in which the features of one class are the Exclusive-OR of a 2-bit binary vector and the features of the second class are the negated features. This paradox, also called the Minsky's Paradox [7], demonstrated that a single perceptron could not correctly classify in this simple scenario. As opposed to this, we show that it is possible to find two optimal linear discriminant functions, given as a pair of straight lines, which is a particular case of the quadratic discriminant function. These classifiers have some advantages over the traditional linear discriminant approaches, such as Fisher's, perceptron learning, and other ones, because the classifier that we obtain is both linear and optimal. Finally, we conclude this introductory section by observing that, to the best of our knowledge, the results of this paper are pioneering. We are unaware of any work that has been done in statistical PR, which investigates the design and use of optimal pairwise linear classifiers.

\section{Pattern Classification}

\subsection{Bayes Decision Theory}

The main goal of PR is to find the class that an object belongs to given its features. In statistical models, the features are represented as random vectors in the domain of the real numbers. In particular, the probability distribution function for a $d$-dimensional random vector $X$ which is normally distributed is

$$
p(X)=\frac{1}{\sqrt{2 \pi}|\Sigma|^{\frac{1}{2}}} e^{-\frac{1}{2}(X-M)^{T} \Sigma^{-1}(X-M)},
$$

where $M$ is the mean vector and $\Sigma$ is the covariance matrix.

Suppose we have two classes, $\omega_{1}$ and $\omega_{2}$, with a priori probability, $P\left(\omega_{1}\right)$ and $P\left(\omega_{2}\right)$. We can write the general inequality specifying the Bayesian classification between two classes as follows:

$$
p\left(X \mid \omega_{1}\right) P\left(\omega_{1}\right) \underset{\omega_{1}}{\stackrel{\omega_{2}}{\lessgtr}} p\left(X \mid \omega_{2}\right) P\left(\omega_{2}\right) .
$$

Equality in (21) represents the discriminant function. Assuming that $\omega_{1}$ and $\omega_{2}$ are represented by normal random vectors with covariance matrices $\Sigma_{1}, \Sigma_{2}$, and mean vectors $M_{1}, M_{2}$, respectively. 
Also, without loss of generality, we assume that $\omega_{1}$ and $\omega_{2}$ have the same $a$ priori probability, 0.5. Taking the logarithm of both sides of (2), the discriminant function is:

$$
\log \frac{\left|\Sigma_{2}\right|}{\left|\Sigma_{1}\right|}-\left[\left(X-M_{1}\right)^{T} \Sigma_{1}^{-1}\left(X-M_{1}\right)\right]+\left[\left(X-M_{2}\right)^{T} \Sigma_{2}^{-1}\left(X-M_{2}\right)\right]=0 .
$$

Consider the two cases in (3). When $\Sigma_{1}=\Sigma_{2}$ the discriminant function is linear [5]. For the case when $\Sigma_{1}$ and $\Sigma_{2}$ are arbitrary, the classifier results in a general equation of second degree in the form of a hyperparaboloid, hyperellipsoid, hypersphere, or a pair of hyperplanes or hyperboloids. Indeed, in our discussion, we are interested in the case when the classifier is a pair of hyperplanes. The classifier is a pair of straight lines for $d=2$.

In the interest of brevity, the proofs of the theorems stated here are omitted. They are found in 12 . Also the bibliography is abbreviated. A more complete bibliography and comparative survey is found in [12], which is the unabridged version of this paper.

As per our survey, the results on pairwise linear classifiers that we present here are the first formal reported results on using such classifiers in any avenue of statistical PR.

\section{Linear Discriminants in Diagonalization}

\subsection{The 2-Dimensional Case}

Diagonalization is the process of transforming a space by performing linear and whitening transformations [4. A brief summary of this strategy can be found in [12] and omitted here in the interest of brevity. Given any arbitrary normal random vectors, $X_{1}$ and $X_{2}$, whose covariance matrices are $\Sigma_{1}$ and $\Sigma_{2}$, we can perform simultaneous diagonalization to obtain normal random vectors, $V_{1}$ and $V_{2}$, whose covariance matrices are diagonal, namely $I$ and $\Lambda$, respectively. In what follows, we assume that the dimension of our problem is $d=2$.

As to be more specific, we assume that after simultaneous diagonalization, the mean vectors and covariance matrices have the form:

$$
M_{1}=\left[\begin{array}{l}
p \\
q
\end{array}\right], M_{2}=\left[\begin{array}{l}
r \\
s
\end{array}\right], \Sigma_{1}=\left[\begin{array}{ll}
1 & 0 \\
0 & 1
\end{array}\right], \text { and } \Sigma_{2}=\left[\begin{array}{cc}
a^{-1} & 0 \\
0 & b^{-1}
\end{array}\right] .
$$

Since we will be, for the present, consistently dealing with two-dimensional vectors we shall assume that the feature vector has the form $X=\left[\begin{array}{l}x \\ y\end{array}\right]$.

For our discussion, we let $X \sim(M, \Sigma)$ denote a normal random vector, $X$, with covariance matrix $\Sigma$ and mean vector $M$. We will now present a linear transformation that will later prove useful in simplifying complex expressions. The transformation is stated more formally in Theorem 1 . 
Theorem 1. Let $X_{1} \sim N\left(M_{1_{X}}, \Sigma_{1}\right)$ and $X_{2} \sim N\left(M_{2_{X}}, \Sigma_{2}\right)$ be two normal random vectors, such that

$$
M_{1_{X}}=\left[\begin{array}{l}
p \\
q
\end{array}\right], M_{2_{X}}=\left[\begin{array}{l}
r \\
s
\end{array}\right], \Sigma_{1}=\left[\begin{array}{ll}
1 & 0 \\
0 & 1
\end{array}\right] \text {, and } \Sigma_{2}=\left[\begin{array}{ll}
a & 0 \\
0 & b
\end{array}\right] .
$$

Vectors $X_{1}$ and $X_{2}$ can be transformed into $Z_{1} \sim N\left(M_{1_{Z}}, \Sigma_{1}\right)$ and $Z_{2} \sim$ $N\left(M_{2_{Z}}, \Sigma_{2}\right)$, where $M_{1_{Z}}=\left[\begin{array}{c}t \\ u\end{array}\right]$ and $M_{2_{Z}}=\left[\begin{array}{l}-t \\ -u\end{array}\right]$.

Theorem 2. Let $X_{1} \sim N\left(M_{1}, \Sigma_{1}\right)$ and $X_{2} \sim N\left(M_{2}, \Sigma_{2}\right)$ be two random vectors, such that:

$$
M_{1}=\left[\begin{array}{l}
r \\
s
\end{array}\right], M_{2}=\left[\begin{array}{l}
-r \\
-s
\end{array}\right], \Sigma_{1}=\left[\begin{array}{ll}
1 & 0 \\
0 & 1
\end{array}\right], \text { and } \Sigma_{2}=\left[\begin{array}{cc}
a^{-1} & 0 \\
0 & b^{-1}
\end{array}\right] .
$$

If $a$ and $b$ are positive real numbers, such that:

$$
a(1-b) r^{2}-\frac{1}{4}(a b-a-b+1) \log a b=(a-1) b s^{2} .
$$

then the optimal classifier obtained by Bayes classification is a pair of straight lines.

Equation (7) is the necessary and sufficient condition that real numbers $a>0, b>0, r$, and $s$, must satisfy in order to yield the optimal linear classifier between two classes represented by normal random vectors with parameters of the form given in (6).

Consider the following: Given positive real numbers $a>0$ and $b>0$, we would like to find real numbers, $r$ and $s$, that satisfy (7). The cases for which it is possible to find real numbers, $r$ and $s$, satisfying (7) are stated below.

Theorem 3. Let $X_{1} \sim N\left(M_{1}, \Sigma_{1}\right)$ and $X_{2} \sim N\left(M_{2}, \Sigma_{2}\right)$ be two normal random vectors, such that

$$
M_{1}=\left[\begin{array}{l}
r \\
s
\end{array}\right], M_{2}=\left[\begin{array}{l}
-r \\
-s
\end{array}\right], \Sigma_{1}=\left[\begin{array}{ll}
1 & 0 \\
0 & 1
\end{array}\right], \text { and } \Sigma_{2}=\left[\begin{array}{ll}
a & 0 \\
0 & b
\end{array}\right] .
$$

For any positive real numbers $a$ and $b$, there exist real numbers $r$ and $s$ only if $a>1$ and $0<b<1$, or $0<a<1$ and $b>1$.

\subsection{The d-Dimensional Case}

In Sect. 3.1 we have presented the necessary and sufficient conditions in which the optimal classifier is a pair of straight lines, for the two dimensional space. We have also shown that for some covariance matrices, it is not possible to find the pairwise linear optimal classifier. We are now interested in finding the cases for the d-dimensional space in which the optimal classifier is a pair of hyperplanes.

Let us consider the more general case for $d>2$. We are required to determine whether it is possible to find a pair of hyperplanes as the optimal Bayes classifier.

This problem can be solved using the result of Theorem 4 below. 
Theorem 4. Let $X_{1} \sim N\left(M_{1}, \Sigma_{1}\right)$ and $X_{2} \sim N\left(M_{2}, \Sigma_{2}\right)$ be two normal random vectors, such that

$$
M_{1}=\left[\begin{array}{c}
m_{11} \\
\vdots \\
m_{1 d}
\end{array}\right], M_{2}=\left[\begin{array}{c}
m_{21} \\
\vdots \\
m_{2 d}
\end{array}\right], \Sigma_{1}=\left[\begin{array}{ccc}
1 & \ldots & 0 \\
\vdots & \ddots & \vdots \\
0 & \ldots & 1
\end{array}\right] \text {, and } \Sigma_{2}=\left[\begin{array}{ccc}
\sigma_{11}^{2} & \ldots & 0 \\
\vdots & \ddots & \vdots \\
0 & \ldots & \sigma_{d d}^{2}
\end{array}\right]
$$

It is not possible to find a pair of hyperplanes as the optimal Bayes classifier.

\section{Special Cases of Linear Discriminant}

In this section we analyze two special cases of diagonal covariance matrices that lead to the optimal linear discriminant function. The necessary and sufficient conditions to achieve a linear classifier are discussed in both cases. The second, and more specific case, is that where the mean vector is the same for the two classes under consideration.

\subsection{Linear Discriminant with Different Means}

Consider two normal random vectors of dimension $d=2$. Using the diagonalization process discussed in [12, any covariance matrices and mean vectors can be converted into the following:

$$
M_{1}=\left[\begin{array}{l}
p \\
q
\end{array}\right], M_{2}=\left[\begin{array}{l}
r \\
s
\end{array}\right], \Sigma_{1}=\left[\begin{array}{ll}
1 & 0 \\
0 & 1
\end{array}\right] \text {, and } \Sigma_{2}=\left[\begin{array}{ll}
a & 0 \\
0 & b
\end{array}\right] .
$$

Starting with normal random vectors and these parameters, we are interested in analyzing linear classifiers for a more particular case.

Theorem 5. Let $X_{1}$ and $X_{2}$ be two normal random vectors with covariance matrices and mean vectors as in (9). It is possible to transform $X_{1}, X_{2}$ into $Z_{1}=A^{T} X_{1}, \quad Z_{2}=A^{T} X_{2}$, respectively, where $A=\left[\begin{array}{cc}a^{-\frac{1}{4}} & 0 \\ 0 & a^{\frac{1}{4}}\end{array}\right]$, and the new covariance matrices and mean vectors have the form:

$$
M_{1_{Z}}=\left[\begin{array}{c}
p^{\prime} \\
q^{\prime}
\end{array}\right], M_{2_{Z}}=\left[\begin{array}{l}
r^{\prime} \\
s^{\prime}
\end{array}\right], \Sigma_{1_{z}}=\left[\begin{array}{cc}
a^{\prime} & 0 \\
0 & b^{\prime}
\end{array}\right], \text { and } \Sigma_{2_{Z}}=\left[\begin{array}{cc}
b^{\prime} & 0 \\
0 & a^{\prime}
\end{array}\right]
$$

only if $b=a^{-1}$.

We now state the conditions necessary to obtain a pair of straight lines when we have the form of (10). 
Theorem 6. Let $X_{1} \sim N\left(M_{1}, \Sigma_{1}\right)$ and $X_{2} \sim N\left(M_{2}, \Sigma_{2}\right)$ be two normal random vectors such that

$$
M_{1}=\left[\begin{array}{l}
p \\
q
\end{array}\right], M_{2}=\left[\begin{array}{l}
r \\
s
\end{array}\right], \Sigma_{1}=\left[\begin{array}{cc}
a^{-1} & 0 \\
0 & b^{-1}
\end{array}\right], \text { and } \Sigma_{2}=\left[\begin{array}{cc}
b^{-1} & 0 \\
0 & a^{-1}
\end{array}\right]
$$

The optimal Bayes classifier is a pair of straight lines when $(p-r)^{2}=(q-s)^{2}$, for $a, b$ any positive real numbers. Moreover, if $M_{1}=\left[\begin{array}{l}r \\ s\end{array}\right]$ and $M_{2}=\left[\begin{array}{l}-r \\ -s\end{array}\right]$, the condition simplifies to $r^{2}=s^{2}$.

Theorem 6] states the necessary and sufficient condition for a pairwise linear classifier between two normal random vectors, with means and covariances of the form given in (11).

We consider now the more general case for $d>2$. We are interested in finding the conditions that guarantee a pairwise linear discriminant function. This is given in Theorem 7 below.

Theorem 7. Let $X_{1} \sim N\left(M_{1}, \Sigma_{1}\right)$ and $X_{2} \sim N\left(M_{2}, \Sigma_{2}\right)$ be two normal random vectors such that

$$
\begin{aligned}
M_{1}= & {\left[m_{11} \ldots m_{1 i} \ldots m_{1 j} \ldots m_{1 d}\right]^{T}, M_{2}=\left[m_{21} \ldots m_{2 i} \ldots m_{2 j} \ldots\right.} \\
\Sigma_{1}= & {\left[\begin{array}{ccccccc}
\sigma_{11}^{2} & 0 & 0 & 0 & 0 & 0 & 0 \\
0 & \ddots & 0 & 0 & 0 & 0 & 0 \\
0 & 0 & \sigma_{i i}^{2} & 0 & 0 & 0 & 0 \\
0 & 0 & 0 & \ddots & 0 & 0 & 0 \\
0 & 0 & 0 & 0 & \sigma_{j j}^{2} & 0 & 0 \\
0 & 0 & 0 & 0 & 0 & \ddots & 0 \\
0 & 0 & 0 & 0 & 0 & 0 & \sigma_{d d}^{2}
\end{array}\right], \text { and } \Sigma_{2}=\left[\begin{array}{ccccccc}
\sigma_{11}^{2} & 0 & 0 & 0 & 0 & 0 & 0 \\
0 & \ddots & 0 & 0 & 0 & 0 & 0 \\
0 & 0 & \sigma_{j j}^{2} & 0 & 0 & 0 & 0 \\
0 & 0 & 0 & \ddots & 0 & 0 & 0 \\
0 & 0 & 0 & 0 & \sigma_{i i}^{2} & 0 & 0 \\
0 & 0 & 0 & 0 & 0 & \ddots & 0 \\
0 & 0 & 0 & 0 & 0 & 0 & \sigma_{d d}^{2}
\end{array}\right] . }
\end{aligned}
$$

The optimal classifier, obtained by Bayes classification, is a pair of hyperplanes when $\left(m_{1 i}-m_{2 i}\right)^{2}=\left(m_{1 j}-m_{2 j}\right)^{2}$, for $k=1, \ldots, d, \sigma_{k k}^{2}$ a positive real number.

\subsection{Linear Discriminant with Equal Means}

In this section, we discuss a particular instance of the problem discussed in Sect. 4.1. Let us consider the generalization of Minsky's paradox, that is, when $M_{1}=M_{2}$. We shall now show that it is always possible to find a pair of straight lines when $M_{1}=M_{2}, \Sigma_{1}=\left[\begin{array}{ll}a & 0 \\ 0 & b\end{array}\right]$, and $\Sigma_{1}=\left[\begin{array}{ll}b & 0 \\ 0 & a\end{array}\right]$, thus re-solving the paradox in the most general case. 
Theorem 8. Let $X_{1} \sim N\left(M_{1}, \Sigma_{1}\right)$ and $X_{2} \sim N\left(M_{2}, \Sigma_{2}\right)$ be two random vectors such that:

$$
M_{1}=M_{2}=\left[\begin{array}{l}
r \\
s
\end{array}\right], \Sigma_{1}=\left[\begin{array}{cc}
a^{-1} & 0 \\
0 & b^{-1}
\end{array}\right], \text { and } \Sigma_{2}=\left[\begin{array}{cc}
b^{-1} & 0 \\
0 & a^{-1}
\end{array}\right]
$$

The optimal classifier obtained by Bayes classification is a pair of straight lines for positive real numbers $a$ and $b$, where $r$ and $s$ are any real numbers.

The power of this will be obvious when the classification results are discussed in a subsequent section.

\section{Classification}

\subsection{The Discriminant Function}

In this section we discuss classification with the linear discriminant functions determined in Sect 4.2, for dimension $d=2$.

The discriminant functions for the cases discussed in Sects. 3.1, 4.1, and 4.2 are quadratic equations that represent pair of straight lines. For the purpose of classification, we need to find one equation for each straight line. This is done by inspection or by solving the quadratic equation in terms of $y$. The second degree polynomial equations, have the following roots:

$$
y_{+}=A_{1} x+B_{1}, \text { and } y_{-}=A_{2} x+B_{2}
$$

Let us consider now the third case discussed in Sect. 4.2, The equation for each straight line can be found as per the following theorem.

Theorem 9. Let $X_{1} \sim N\left(M_{1}, \Sigma_{1}\right)$ and $X_{2} \sim N\left(M_{2}, \Sigma_{2}\right)$ be two random vectors such that

$$
M_{1}=M_{2}=\left[\begin{array}{l}
r \\
s
\end{array}\right], \Sigma_{1}=\left[\begin{array}{cc}
a^{-1} & 0 \\
0 & b^{-1}
\end{array}\right] \text {, and } \Sigma_{2}=\left[\begin{array}{cc}
b^{-1} & 0 \\
0 & a^{-1}
\end{array}\right] \text {. }
$$

The equations of the linear discriminant functions, i.e. the optimal classifiers, are

$$
y_{+}=-x+(r+s) \text { and } y_{-}=x+(s-r)
$$

Special cases of the discriminant functions for the distributions discussed in Sects. 3.1 and 4.1, are found in [12]. 


\section{Simulation Results}

In this section we present some examples illustrating the different cases discussed in previous sections. In all of the examples we have chosen the dimension $d=2$ and two classes, $\omega_{1}$ and $\omega_{2}$. We also discuss the empirical results obtained after testing the linear classifier with 100 points for each class generated randomly using the maximum likelihood approach in estimating the parameters [13], assuming that they are of the form found in the respective cases.

The two classes, $\omega_{1}$ and $\omega_{2}$, are represented by two normal random vectors $X_{1} \sim N\left(M_{1}, \Sigma_{1}\right), X_{2} \sim N\left(M_{2}, \Sigma_{2}\right)$. We used one instance for each of the three cases and generated a set of 100 normal random points, in order to test the accuracy of the classifiers.

In the first test (referred to as DD, whose plot is given in [12]) we considered the pairwise linear discriminant function in diagonalization. We used the following covariance matrices and mean vectors (estimated from 100 training samples) to yield the respective classifier:

$$
M_{1} \approx\left[\begin{array}{l}
1.0342 \\
1.8686
\end{array}\right], M_{2} \approx\left[\begin{array}{l}
-1.0342 \\
-1.8686
\end{array}\right], \Sigma_{1}=\left[\begin{array}{ll}
1 & 0 \\
0 & 1
\end{array}\right], \Sigma_{2} \approx\left[\begin{array}{cc}
.4599 & 0 \\
0 & 2.8232
\end{array}\right] .
$$

The accuracy of the classifier was $98 \%$ for $\omega_{1}$ and $99 \%$ for $\omega_{2}$. The power of the scheme is obvious!

In the second test (referred to as DM, whose plot is also found in [12]) we considered the pairwise linear discriminant with different means. The following estimated covariance matrices and mean vectors were obtained by using 100 training samples.

$$
M_{1} \approx\left[\begin{array}{c}
-.9555 \\
.9555
\end{array}\right], M_{2} \approx\left[\begin{array}{c}
.9555 \\
-.9555
\end{array}\right], \Sigma_{1} \approx\left[\begin{array}{cc}
1.808 & 0 \\
0 & .3188
\end{array}\right], \Sigma_{2} \approx\left[\begin{array}{cc}
.3188 & 0 \\
0 & 1.808
\end{array}\right]
$$

Using the above parameters, the pairwise linear classifier was derived. The plot of the points and the linear discriminant function are shown in 12 . The accuracy of the classifier was $91 \%$ for $\omega_{1}$ and $95 \%$ for $\omega_{2}$.

To show the power of the scheme, we also tested our results for the case of the pairwise linear classifier with equal means (EM) for the generalized Minsky's Paradox. By using 100 training samples generated with equal means but mirrored covariances, we obtained the following estimated covariance matrices and mean vectors:

$$
M_{1}=M_{2} \approx\left[\begin{array}{l}
5.9841 \\
6.1766
\end{array}\right], \Sigma_{1} \approx\left[\begin{array}{cc}
.0904 & 0 \\
0 & 12.752
\end{array}\right], \Sigma_{2} \approx\left[\begin{array}{cc}
12.752 & 0 \\
0 & .0904
\end{array}\right] .
$$

The plot of the points and the linear discriminant function from these estimates is given in Fig. 11 The accuracy of the classifier was $96 \%$ for $\omega_{1}$ and $90 \%$ for $\omega_{2}$. The accuracy is very high in this case, despite the fact that the classes overlap and the discriminant functions are pairwise linear. The power of the results presented is obvious. 


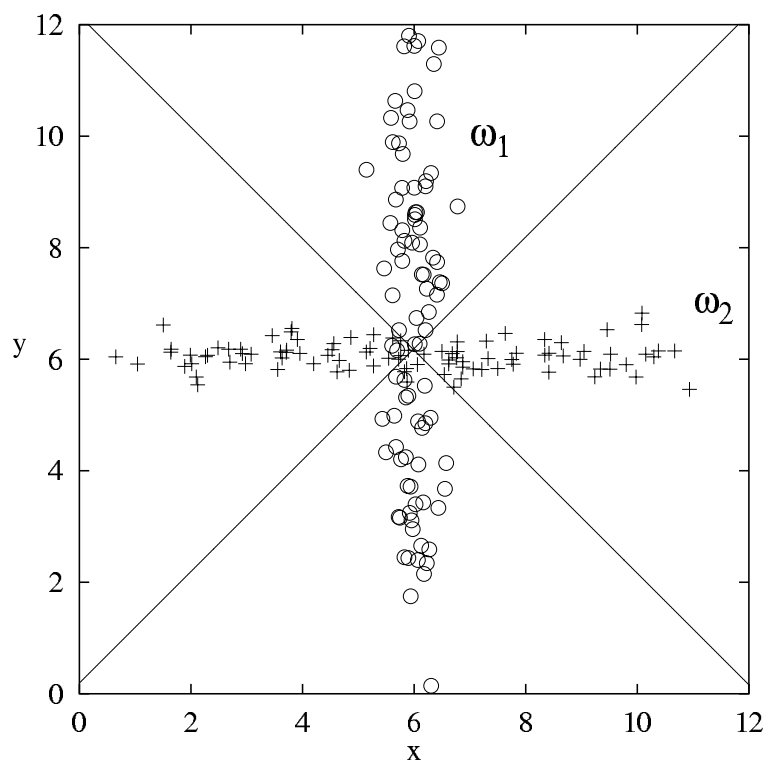

Fig. 1. Example of pairwise linear discriminant with equal means for the case described in Theorem 8. This represents the resolution of the general case of Minsky's Paradox.

\section{Conclusions}

In this paper we have shown the problem of determining pairwise linear classifiers for the case of normally distributed classes. We have shown that, contrary to what is known, it is possible to find the optimal linear discriminant function even though that the covariance matrices are different. In all the cases discussed here, the functions obtained are pairs of straight lines, which are a particular case of the second degree general equation.

By a formal procedure, we have determined the conditions for these particular discriminant functions in three cases. The first case occurs after diagonalization. We have explicitly derived the necessary and sufficient conditions for the covariance matrices and the mean vectors so as to yield a pair of straight lines for the optimal classifier. We have also shown that it is impossible to find a pair of hyperplanes as the optimal Bayes classifier in the d-dimensional case.

The second case is when we have particular forms in the two diagonal covariance matrices.

In the third case, assuming equal means, we have found that it is always possible to obtain a pair of straight lines when we have covariance matrices with the same form as found in the second case, thus re-solving Minsky's paradox!

The results derived in the paper have also been experimentally verified. The empirical results obtained show that the accuracy of the classifier is very high. 
This is understandable since the classifier is optimal. The degree of this accuracy is even more amazing when we recognize that we are dealing with a linear discriminant function for classes which are significantly overlapping.

Acknowledgements : The work of the first author was partially supported by Departamento de Informática, Universidad Nacional de San Juan, Argentina, and the work of the second author was partially supported by NSERC, the National Science and Engineering Research Council of Canada.

\section{References}

1. M. Aladjem. Linear Discriminant Analysis for Two Classes Via Removal of Classification Structure. IEEE Trans. Pattern Analysis and Machine Intelligence, 19(2):187-192, 1997.

2. P. Devijver and J. Kittler. Pattern Recognition: A Statistical Approach. PrenticeHall, 1982.

3. R. Duda and P. Hart. Pattern Classification and Scene Analysis. John Wiley and Sons, Inc., 1973.

4. K. Fukunaga. Introduction to Statistical Pattern Recognition. Academic Press, 1990.

5. K. Fukunaga. Statistical Pattern Recognition. Handbook of Pattern Recognition and Computer Vision, pages 33-60, 1993.

6. T. M. Ha. Optimum Decision Rules in Pattern Recognition. Advances in Pattern Recognition, SSPR'98 - SPR'98, pages 726-735, 1998.

7. M. Minsky. Perceptrons. MIT Press, 2nd edition, 1988.

8. A. Rao, D. Miller, K. Rose, , and A. Gersho. A Deterministic Annealing Approach for Parsimonious Design of Piecewise Regression Models. IEEE Trans. Pattern Analysis and Machine Intelligence, 21(2):159-173, 1999.

9. S. Raudys. Linear Classifiers in Perceptron Design. Proc. 13th ICPR, Track D, Wien, 1996.

10. S. Raudys. On Dimensionality, Sample Size, and Classification Error of Nonparametric Linear Classification. IEEE Trans. Pattern Analysis and Machine Intelligence, 19(6):667-671, 1997.

11. B. Ripley. Pattern Recognition and Neural Networks. Cambridge Univ. Press, 1996.

12. L. Rueda and B.J. Oommen. On Optimal Pairwise Linear Classifiers. Unabridged version of this paper. Submitted for Publication.

13. R. Schalkoff. Pattern Recognition: Statistical, Structural and Neural Approaches. John Wiley and Sons, Inc., 1992. 